\title{
Mapping of Chromatic-Dispersion Distribution Along Optical Fibers With 20-m Spatial Resolution
}

\author{
Kwang-Yong Song, Miguel González Herráez, and Luc Thévenaz
}

\begin{abstract}
The accurate mapping of chromatic-dispersion distribution along single-mode optical fibers with the best spatial resolution ever reported is experimentally demonstrated. The method consists of a new way of probing four-wave-mixing (FWM) efficiency along the fiber using Brillouin-optical-time-domain analysis (BOTDA). The new setup eliminates many noise problems found in previous configurations, allowing a tenfold improvement in the spatial resolution of this kind of measurements. Resolutions of $20 \mathrm{~m}$ in a 1.1-km standard fiber and $150 \mathrm{~m}$ in a $6.7-\mathrm{km}$ dispersion-shifted fiber (DSF), respectively, were achieved.
\end{abstract}

Index Terms-Brillouin scattering, nonlinear optics, opticalfiber dispersion, optical-fiber measurements.

\section{INTRODUCTION}

$\mathbf{P}$ RECISE information about chromatic-dispersion distribution along optical fibers has been shown to be beneficial in experiments related to massive wavelength-division multiplexing (WDM) and dispersion-managed soliton transmission [1]. Additionally, recent studies concerning fiber-optic parametric amplifiers and wavelength converters show that dispersion fluctuations might be strongly detrimental in these devices if they are uncontrolled [2], or highly beneficial if they are adequately designed and tailored [3]. As a result, several methods for mapping the variation of the chromatic dispersion along the fiber have already been reported in the literature [4]-[6]. In particular, the method reported in [6] using four-wave mixing (FWM) and Brillouin-optical-time-domain analysis (BOTDA) has shown explicit advantages over the others because it can be used in the wavelength region of small anomalous dispersion, where its applications are extremely interesting.

As for the spatial resolution of the measurement, the best resolutions experimentally achieved up to now have been $200 \sim 300 \mathrm{~m}$ [6]. These figures might be adequate for the characterization of soliton-based fiber links, but they are still too large for nonlinear devices (namely parametric amplifiers and wavelength converters) requiring high-resolution dispersion mapping in short-length fibers of only $1-2 \mathrm{~km}$. Additionally, these devices always work in the region of small

Manuscript received May 18, 2005; revised July 20, 2005.

K.-Y. Song was with the Nanophotonics and Metrology Laboratory, Ecole Polytechnique Fédérale de Lausanne, CH-1015, Lausanne, Switzerland. He is now with the Department of Electronic Engineering, The University of Tokyo, Tokyo 113-8656 Japan (e-mail: songky@kaist.ac.kr).

M. González Herráez is with the Nanophotonics and Metrology Laboratory, Ecole Polytechnique Fédérale de Lausanne, CH-1015, Lausanne, Switzerland, on leave from the Department of Electronics, University of Alcalá, Madrid, 28871 Spain (e-mail: miguelg@depeca.uah.es).

L. Thévenaz is with the Nanophotonics and Metrology Laboratory, Ecole Polytechnique Fédérale de Lausanne, CH-1015, Lausanne, Switzerland (e-mail: luc.thevenaz@epfl.ch).

Digital Object Identifier 10.1109/JLT.2005.858240 anomalous dispersion of the fiber, so our method seems specially suited to cover this range of applications. Although some of previous works have reported better $(\sim 100 \mathrm{~m})$ spatial resolution using the bidirectional optical-time-domain-reflectometer (OTDR) technique [7], the obtained measurement accuracy depended highly on the availability of accurate information of certain fiber parameters, such as the wavelength dependence of the mode-field diameter. This fact significantly limits the practicality of this method, in favor of a more direct determination of the chromatic dispersion as a function of the position.

In this paper, we present a record performance in the dispersion mapping of optical fibers using a substantially improved experimental configuration of the method reported in [6]. We achieve spatial resolutions of $20 \mathrm{~m}$ in a standard fiber and $150 \mathrm{~m}$ in a dispersion-shifted fiber (DSF), which are, to our knowledge, the best resolutions ever reported. We also discuss some limiting factors in spatial-resolution improvement using this technique.

\section{PRINCIPLE}

When two moderately powerful pump waves $\left(f_{1}>f_{2}\right)$ copropagate along an optical fiber, the process of FWM causes the generation of two new frequencies named Stokes $\left(f_{\mathrm{S}}\right)$ and anti-Stokes $\left(f_{\mathrm{A}}\right)$ waves, depending on their energy content. The process can be viewed as the collapse of two pump photons at $f_{1}\left(f_{2}\right)$ to generate a photon at $f_{2}\left(f_{1}\right)$ and another photon at $f_{\mathrm{A}}=2 f_{1}-f_{2}\left(f_{\mathrm{S}}=2 f_{2}-f_{1}\right)$, thus following energyconservation rules. The efficiency of the process is controlled by the phase mismatch $\Delta \beta \cdot L$ between the pump photons that collapse and the new photons generated. For the anti-Stokes wave, this phase mismatch is governed by $\Delta \beta=2 \beta\left(\lambda_{1}\right)-$ $\beta\left(\lambda_{2}\right)-\beta\left(\lambda_{\mathrm{A}}\right)$, where $\beta$ is the propagation constant. Whenever $\Delta \beta=0$, all the generated contributions add coherently and the power of the FWM product grows with the square of the fiber distance. However, when $\Delta \beta \neq 0$ the phase mismatch grows linearly with the distance, and the FWM contributions generated along the fiber add constructively and destructively, in an alternative way. In this last case, the power of the new waves oscillates along the fiber with a spatial frequency given by $\Delta \beta / 2 \pi$. If the wavelength difference $(\Delta \lambda)$ between the two pumps is small and the nonlinear phase-shift effect is ignored (this effect is negligible with the usual FWM-pump levels used in our experiment), the term $\Delta \beta$ is directly related to the local chromatic dispersion $(D)$ of the fiber by the following equation [4], [6]

$$
\Delta \beta=\frac{2 \pi}{\Lambda}=\frac{2 \pi \cdot c}{\lambda^{2}} \cdot D \cdot \Delta \lambda^{2}
$$


where $c$ is the speed of light, $\Lambda$ is the spatial oscillation period, and $\lambda$ is the wavelength of the FWM pumps. As we can see, perfect phase matching only occurs when the chromatic dispersion at the pump wavelength is zero, or equivalently, when the pump is tuned to the zero-dispersion wavelength of the fiber. In our measurements, we tune the pump wavelength to a spectral region in which the fiber has a nonnegligible value for chromatic dispersion, so as to have a phase-mismatched interaction. The power of anti-Stokes wave $P_{\mathrm{A}}$ as a function of the position $z$ is expressed as

$P_{\mathrm{A}}(z)=8 \cdot\left(\frac{n_{2} \cdot \lambda}{c \cdot A_{\mathrm{eff}}}\right)^{2} \cdot \frac{P_{1}^{2} \cdot P_{2}}{\Delta \lambda^{4} \cdot D^{2}} \cdot \sin ^{2}\left(\frac{\pi \cdot c}{\lambda^{2}} \cdot D \cdot \Delta \lambda^{2} \cdot z\right)$

where $P_{1}\left(P_{2}\right)$ is the local power of $f_{1}\left(f_{2}\right), A_{\text {eff }}$ is the effective area covered by the fundamental fiber mode, and $n_{2}$ is the nonlinear refractive index of silica. A similar expression can be found for the Stokes wave by simply exchanging the subscripts 1 to 2 and $\mathrm{A}$ to $\mathrm{S}$ in (2) and in the expression of the phase mismatch given above. The basic idea of the local measurement of chromatic dispersion is to retrieve the power of the anti-Stokes (Stokes) wave $P_{\mathrm{A}}\left(P_{\mathrm{S}}\right)$ along the fiber using BOTDA. Since the local oscillation period is directly related to the local value of the chromatic-dispersion coefficient, a measure of this power distribution and a simple spectrogram analysis should give us a simple and precise way of estimating $D(z)$. In comparison to the OTDR-based measurement [4], the BOTDA method allows the use of low-power FWM pumps, thus avoiding interference from other nonlinear effects in the measurement, namely modulation instability (MI) and Raman. In particular, since the FWM-pump powers are well below the MI threshold, our method might be used in fibers exhibiting anomalous dispersion at the pump wavelengths. This is the most interesting region to measure both in soliton-based fiber links and in fibers used for nonlinear devices like parametric amplifiers and wavelength converters.

For the BOTDA configuration, a spectrally narrow pump pulse needs to be launched into the fiber under test in the opposite direction and tuned to a frequency that would cause Brillouin gain over the analyzed FWM product. When the antiStokes wave is used as a probe signal, the optical frequency of the Brillouin-pump pulse has to be approximately $f_{\mathrm{A}}+\nu_{\mathrm{B}}$, with $\nu_{\mathrm{B}}$ being the Brillouin shift of the fiber. In this case, the signal obtained at the BOTDA fiber end (tuned at $f_{\mathrm{A}}$ ) can be expressed as

$$
\Delta P_{\mathrm{A}}=\frac{g_{\mathrm{B}}}{A_{\mathrm{eff}}} P_{\mathrm{A}}(z) P_{\mathrm{P}}(z) \Delta z
$$

where $g_{\mathrm{B}}$ is the peak Brillouin linear gain, $P_{\mathrm{P}}$ is the power of the Brillouin pump, and $\Delta z$ is the pump pulsewidth, respectively. The oscillation of $P_{\mathrm{A}}$ is translated into a temporal oscillation in the detected BOTDA signal, from which the local dispersion can be retrieved through a simple spectrogram analysis [6].

The measurement principle of a typical BOTDA setup is shown in Fig. 1(a). Normally, a continuous-wave (CW) probe

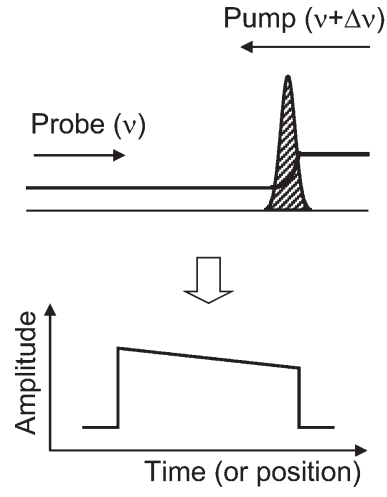

(a)

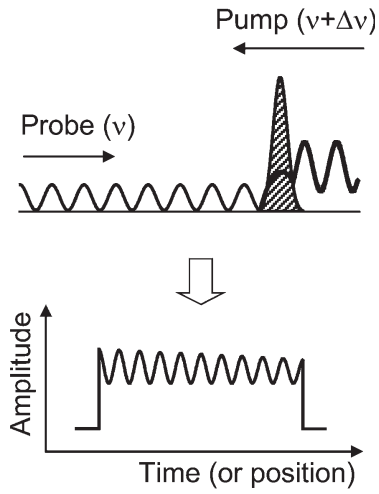

(b)
Fig. 1. Schematics of the measurement principles used in (a) a typical BOTDA setup and (b) a dispersion-mapping experiment using FWM and BOTDA (this work)

wave and a pump pulse tuned at the correct frequency counterpropagate for stimulated Brillouin interaction. The frequency difference $\Delta \nu$ between the pump and the probe waves is swept around $\nu_{\mathrm{B}}$, and the Brillouin gain as a function of the position is recorded for each frequency step. Our measurement principle is shown in Fig. 1(b). The acquisition is done exactly in the same way as in the usual BOTDA setup, but now the probe signal is the FWM signal, which oscillates along the fiber. In the analysis step, the frequency difference between pump and probe $(\Delta \nu)$ that gives the maximum gain is determined for each position. While in the classical BOTDA setup this is the essential aim of the measurement, in our case, we are interested in reconstructing the FWM signal, which is proportional to the maximum measured signal for each position. From this, the spatial oscillation period of the FWM-probe wave can be retrieved as a function of the position, and hence, the position-resolved value of the chromatic dispersion can be derived.

As stated previously, the spatial period in each position is evaluated using a spectrogram analysis. In this spectrogram analysis, a portion of the signal around the point of interest (analysis window) is Fourier transformed and the dominantfrequency component is regarded as the spatial oscillation frequency. The spatial resolution of the measurement is thus basically determined by the window size of the spectrogram analysis. It has been reported that to keep the uncertainty of the chromatic-dispersion measurement below 5\% [4], [6], the size of the analysis window has to be at least that of a single period of oscillation. Therefore, with this restriction, the spatial oscillation period $\Lambda$ of the BOTDA signal can be directly regarded as the spatial resolution of the measurement. Thus, to achieve a better resolution, $\Delta \lambda$ has to be increased, but this comes at the expense of a lower amplitude of the oscillations [see (2)]. Obviously, the time-base resolution of the acquisitions must be sufficiently small to ensure that the measured waveform adequately represents the FWM distribution. In the case of large noise, the size of the analysis window needs to be increased more than $\Lambda$ to assure the accuracy of the measurement. In our experiment, the measurement for each fiber is performed twice (one from each side), and the difference of the results is regarded as an 


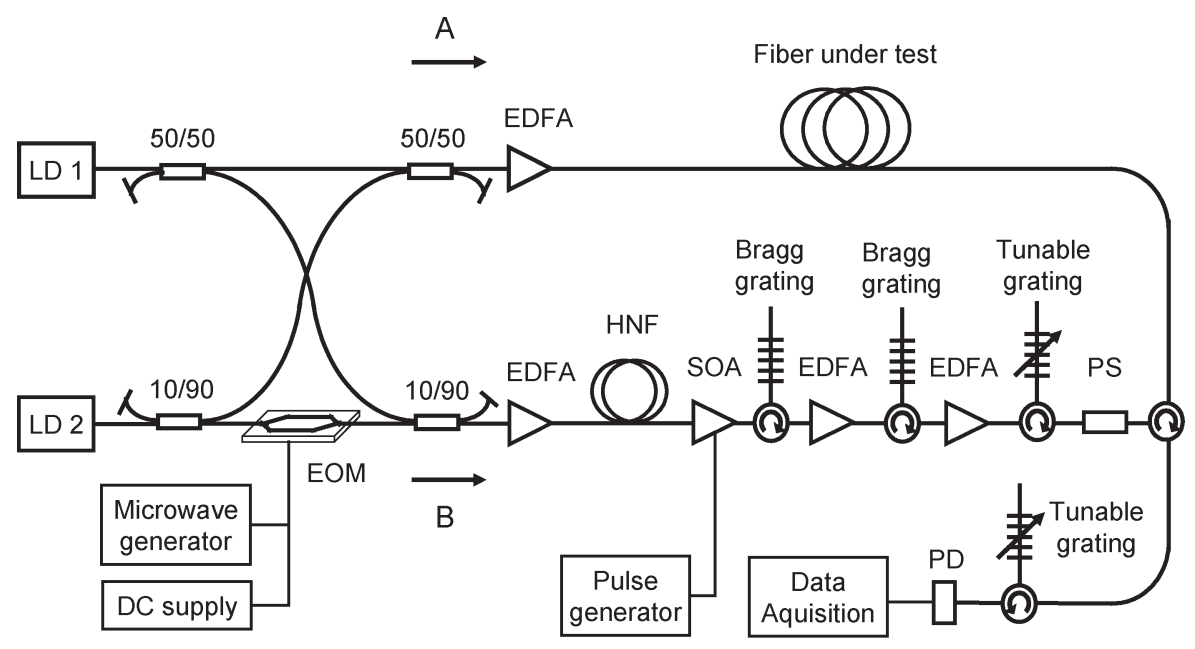

(a)

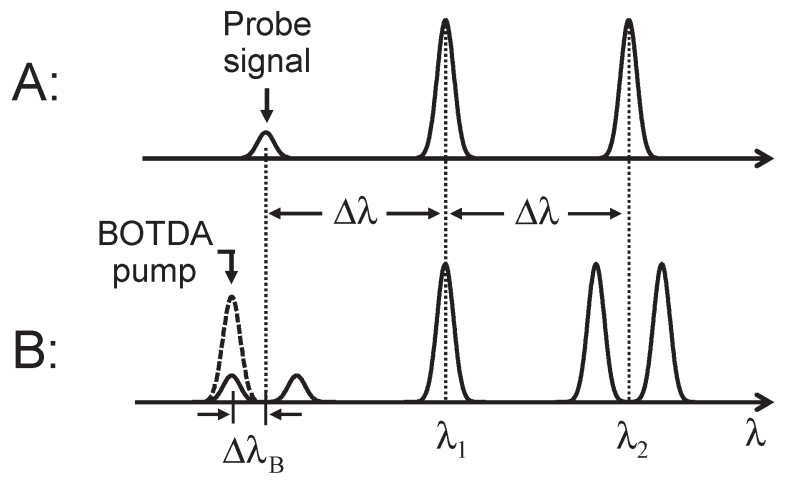

(b)

Fig. 2. (a) Experimental setup. (b) Wavelength arrangement for the FWM pumps (A) and the Brillouin pump (B), respectively. $\Delta \lambda_{\mathrm{B}}$ is the Brillouin shift of the fiber.

estimation of the uncertainty of measurement. We determined the size of the analysis window that can keep the uncertainty below $5 \%$.

An important question is the choice of Brillouin-pump pulsewidth. While $\Delta z$ is smaller than $\Lambda / 2$, longer pulses deliver a cleaner signal with larger amplitude. However, if $\Delta z$ becomes larger than $\Lambda / 2$, the contrast of the oscillations gradually vanishes, resulting in a loss of accuracy. Therefore, the optimum width of the Brillouin-pump pulse is half of the oscillation period, in agreement with Nyquist's theorem.

\section{EXPERIMENT AND RESULTS}

The most critical point in this measurement method is how to generate the Brillouin pump from the two FWM pumps so as to guarantee that the frequency difference between the BOTDA pump and the FWM probe remains tuned to $\nu_{\mathrm{B}}$ with a stability not exceeding a few megahertz. This was already solved in [6] by using DC-suppressed external modulation of one of the two FWM pumps at $\nu_{\mathrm{B}} / 2$ and FWM in a highly nonlinear semiconductor optical amplifier (SOA) to generate the Brillouin pump. Although this method ensured perfect frequency stability between the pump and probe beams, one of the FWM pumps launched into the fiber under test actually consisted of a pair of sidebands, leading to nonlinear interactions between the modu- lation sidebands and producing some noise in the detected signal. Our present setup eliminates this problem by introducing different wavelength arrangements for the FWM and BOTDA pumps, while at the same time keeping the stable frequency difference between the Brillouin pump and the FWM probe. This results in a tenfold improvement in the best resolution reported for the measurement of standard fibers (from 200 to $20 \mathrm{~m}$ ) and in a twofold improvement in the best resolution reported in DSFs (from 300 to $150 \mathrm{~m}$ ).

The present experimental setup and the wavelength arrangement are depicted in Fig. 2(a) and (b), respectively. As shown, the wavelength arrangement used for the FWM pumps (direction A) and the Brillouin pump (direction B) are clearly different. This is the main difference with the setup described in [6], and also the main reason for the dramatic increase in the obtained resolution. In our new configuration, we apply the modulation of the wavelength component $\lambda_{2}$ only to the Brillouin-pump wave, while the FWM pumps are composed of two spectrally pure wavelengths coming directly from two lasers as shown in Fig. 2(b). This feature offers a significant suppression of noise in the probe wave. Additionally, the separation of the two strong pump waves at $\lambda_{2}$ in the Brillouinpump direction (B) is doubled from the former $\Delta \lambda_{\mathrm{B}}$ to $2 \Delta \lambda_{\mathrm{B}}$, which enables a substantially better filtering of the Brillouinpump pulse. 


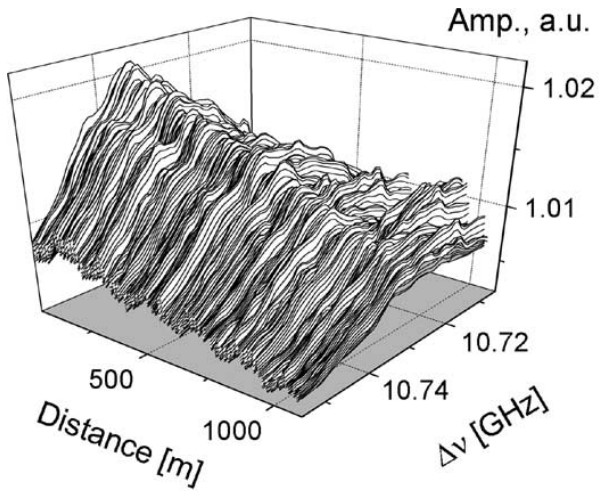

(a)

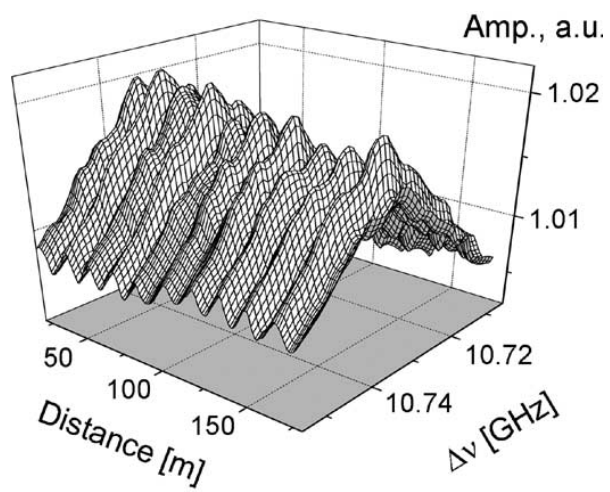

(b)

Fig. 3. (a) Three dimensional (3-D) plot of the measurement result using a 1.1-km standard fiber and (b) its magnified view showing the spatial oscillation of Brillouin gain. $\lambda_{1}: 1558 \mathrm{~nm} . \Delta \lambda: 5.5 \mathrm{~nm} . \Delta z: 8 \mathrm{~m}$.

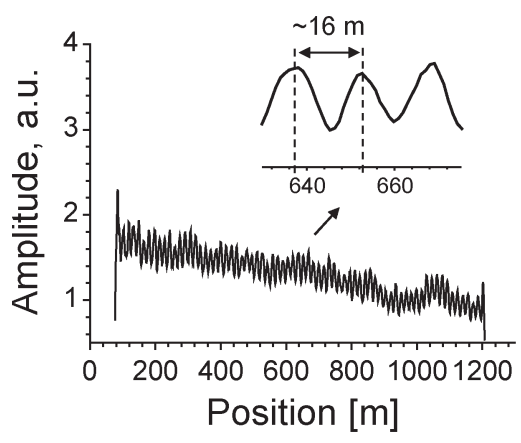

(a)

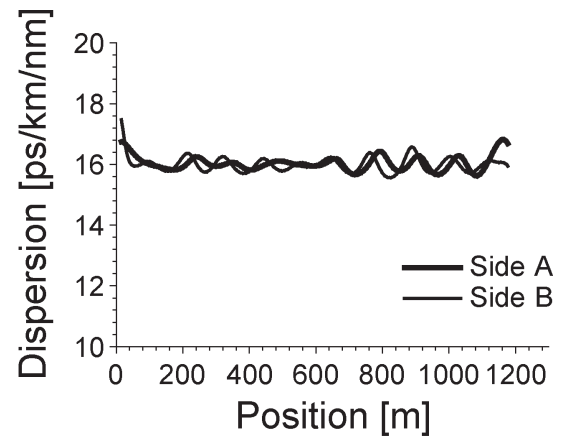

(b)

Fig. 4. (a) Trace of maximum Brillouin gain in a standard fiber, showing an oscillation period of about $16 \mathrm{~m}$. (b) Dispersion map calculated from the measured traces of both sides. The spatial resolution (analysis window) is 20 m. $\lambda_{1}: 1558 \mathrm{~nm} . \Delta \lambda: 5.5 \mathrm{~nm} . \Delta z: 8 \mathrm{~m}$.

The output of two tunable laser diodes (LDs) were combined for use as the FWM-pump waves, and each spectral component was amplified up to $50 \mathrm{~mW}$ using a high-power erbium-doped fiber amplifier (EDFA) in the direction A shown in Fig. 2(a).

In the direction of the Brillouin pump (B), the output of LD2 was modulated using an electrooptic modulator (EOM) to produce two first-order sidebands separated by approximately $2 \Delta \lambda_{\mathrm{B}}$. The carrier wave was suppressed by controlling the DC bias voltage delivered to the EOM with a feedback circuit [8]. The modulated wave and the output of LD1 were combined and amplified using another EDFA up to $30 \mathrm{~mW}$ for each spectral component. The initial seed for the Brillouin-pump pulse was generated from the longer wavelength part of the two sidebands at $\lambda_{2}$ (together with the pump at $\lambda_{1}$ ) by FWM in a 500-m highly nonlinear fiber (HNF) and an SOA. The HNF was inserted to obtain a larger amplitude from the Brillouin pump by increasing the FWM efficiency.

The Brillouin-pump pulses were generated by gating the SOA using an electric pulse train, and they were subsequently filtered and amplified using Bragg gratings and EDFAs to increase the power up to a few hundred milliwatts. The Brillouin-pump pulses were launched into the fiber in the counterpropagating direction to produce local Brillouin gain on the FWM-probe signal along the fiber. The differences in the probe power $\left(\Delta P_{\mathrm{A}}\right)$ were measured using a $125 \mathrm{-MHz}$ photodiode (PD) and data were acquired using a digital oscilloscope synchronized with the Brillouin-pump pulse. The width of the pump pulse was set to approximately half of the oscillation period of the probe signal, so as to maximize the contrast. The polarization of the pump pulse was scrambled using a polarization scrambler (PS) and the acquired traces were averaged several thousand times, ensuring that no polarization-dependent gain was recorded in the trace. The frequency separation between the Brillouin-pump pulse and the probe signal was swept over a 100-MHz range around the $\nu_{\mathrm{B}}$ of the measured fiber and the gain was recorded for each position of the fiber.

Fig. 3(a) shows the three-dimensional (3-D) plot of the measurement result of a standard fiber with a chromatic dispersion of about $16 \mathrm{ps} / \mathrm{km} / \mathrm{nm}$. The length of the fiber was $1.1 \mathrm{~km}$ and the average $\nu_{\mathrm{B}}$ was $10.736 \mathrm{GHz}$. The wavelength of analysis $\lambda_{1}$ was $1558 \mathrm{~nm}$ and $\Delta \lambda$ was set to $5.5 \mathrm{~nm}$. Fig. 3(b) is a magnified view of the plot, where the spatial oscillations of the signal are clearly seen.

The maximum Brillouin gain at each position was retrieved from the 3-D data and depicted as a trace in Fig. 4(a), where the inset shows the magnified view of the oscillation period of about $16 \mathrm{~m}$. We made measurements from both sides of the fiber and performed the spectrogram analysis using a window size of $20 \mathrm{~m}$, which corresponds to the spatial resolution of the measurement. The resultant dispersion map is depicted in Fig. 4(b). The results from both sides were fully consistent and their difference was less than $\pm 4 \%$. A notable feature in these results is the appearance of oscillations in the final 


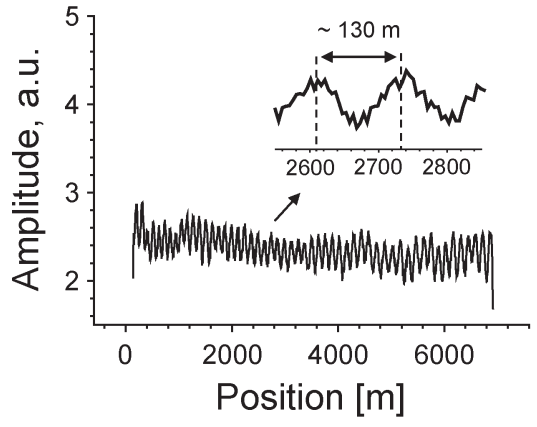

(a)

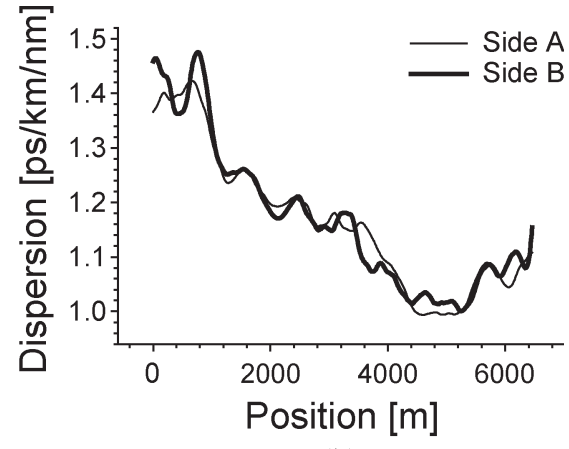

(b)

Fig. 5. (a) Trace of maximum Brillouin gain in a DSF, showing an oscillation period of about $130 \mathrm{~m}$. (b) Dispersion map calculated from the measured traces of both sides. The analysis window is $150 \mathrm{~m} . \lambda_{1}: 1559.4 \mathrm{~nm} . \Delta \lambda: 7.35 \mathrm{~nm} . \Delta z: 65 \mathrm{~m}$.

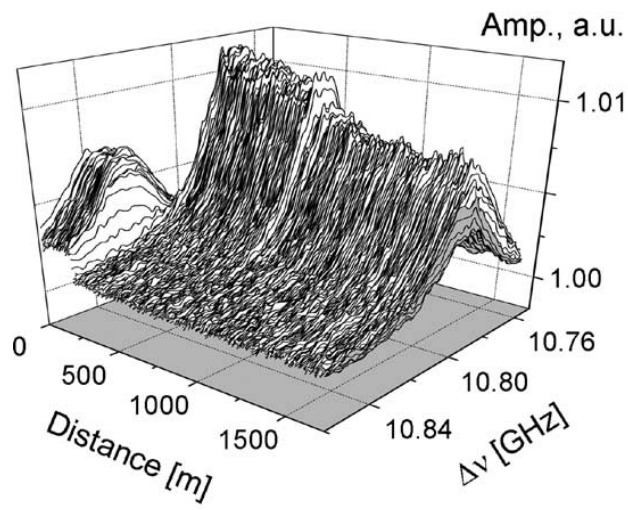

(a)

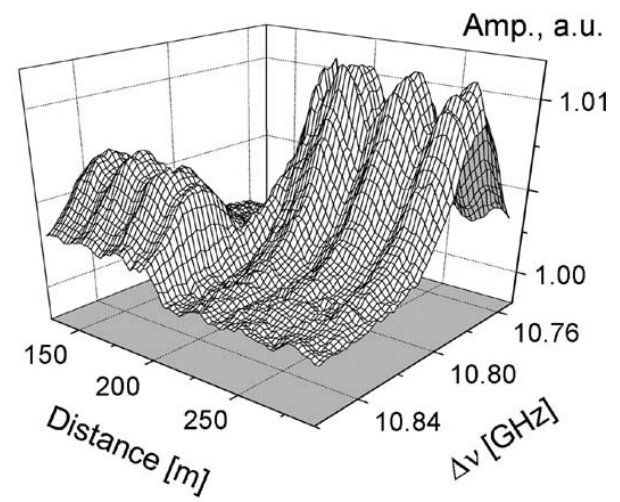

(b)

Fig. 6. (a) Three dimensional (3-D) plot of the measurement result with concatenation of two different fibers with lengths of $200 \mathrm{~m}$ and $1530 \mathrm{~m}$, respectively, and (b) the magnified view showing the spatial oscillation of Brillouin gain at different $\Delta \nu$ 's. $\lambda_{1}: 1558 \mathrm{~nm} . \Delta \lambda: 4.75 \mathrm{~nm} . \Delta z: 10 \mathrm{~m}$.

dispersion map retrieved from both sides. These oscillations have short periods (this is the reason why we believe they were unobserved before) and they might be due to feedback effects in the fabrication process. Similar results were observed in other tested fibers.

Fig. 5 shows the measurement result of a DSF with a chromatic dispersion of about $1.2 \mathrm{ps} / \mathrm{km} / \mathrm{nm}$ at the measured wavelength. The fiber length was $6.7 \mathrm{~km}$ and the average $\nu_{\mathrm{B}}$ was $10.41 \mathrm{GHz}$. The analysis wavelength $\lambda_{1}$ was $1559.4 \mathrm{~nm}$, and $\Delta \lambda$ was set to $7.35 \mathrm{~nm}$. The maximum Brillouin gain at each position is depicted in Fig. 5(a), where the inset shows the magnified view of the oscillation period of about $130 \mathrm{~m}$. Fig. 5(b) shows the calculated dispersion map using traces from both fiber ends. The spatial resolution of the spectrogram in this case is $150 \mathrm{~m}$. The maximum difference between both sides is about $\pm 5 \%$. In the case of the DSF, the signal suffered larger noise that seemed to originate from the onset of spontaneous Brillouin scattering of the FWM-pump waves.

This measurement scheme can be applied to a structure composed of several kinds of fibers having different dispersion and $\nu_{\mathrm{B}}$ 's. As an example of the concatenated measurement of different fibers, we spliced two kinds of standard fibers with the zero-dispersion wavelength around $1300 \mathrm{~nm}$ and performed the measurement. Fig. 6(a) shows the 3-D plot of the result, where two distinct Brillouin-gain curves are observed at the position of each fiber. The lengths and the $\nu_{\mathrm{B}}$ 's of the fibers were $200 \mathrm{~m}$ with $10.833 \mathrm{GHz}$ and $1530 \mathrm{~m}$ with $10.79 \mathrm{GHz}$, respectively.
The wavelength of analysis $\lambda_{1}$ was $1558 \mathrm{~nm}$ and $\Delta \lambda$ was set to $4.75 \mathrm{~nm}$. Fig. 6(b) is a magnified view of the plot, where the spatial oscillation of the signal is clearly seen centered at each $\nu_{\mathrm{B}}$ 's.

The resultant maximum Brillouin gain is depicted in Fig. 7(a). The inset shows the trace oscillation around the splice point of the two fibers, where two different periods of 20 and $26 \mathrm{~m}$ are distinguished for each. Fig. 7(b) shows the calculated dispersion map with a spectrogram window of $30 \mathrm{~m}$, where the abrupt change of dispersion at the splice point is clearly seen.

Since the resolution is simply determined by the oscillating period of the trace, higher resolution measurements can be achieved if $\Delta \lambda$ is increased according to (1). However, more powerful FWM pumps and/or a Brillouin pump are required to maintain the amplitude of the signal given by (2). Therefore, we can think of a few limiting factors preventing further improvement of the spatial resolution:

1) the onset of spontaneous Brillouin scattering from the FWM-pump power that results in FWM-pump depletion and a large amount of noise;

2) the gradual polarization misalignment of the FWM-pump waves due to the residual birefringence in the fiber for larger $\Delta \lambda$, giving rise to a decrease of the FWM efficiency;

3) the MI that depletes and limits the amplitude of the Brillouin-pump pulse. 


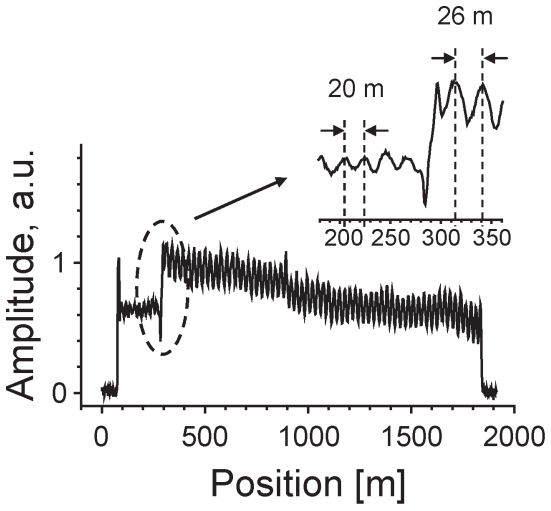

(a)

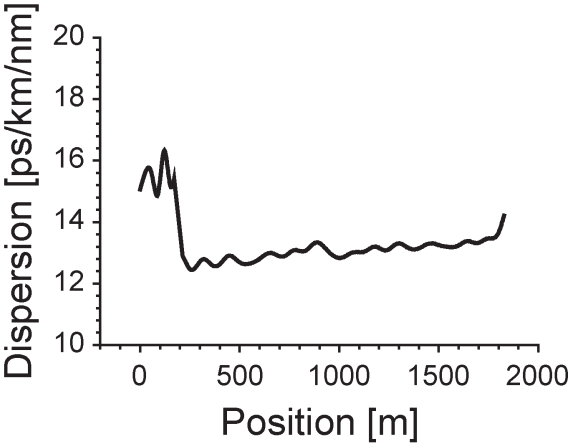

(b)

Fig. 7. (a) Trace of maximum Brillouin gain for the concatenation of two different fibers. The inset shows two spatial periods around the splice point. (b) Dispersion map calculated from the measured trace of the concatenated fibers. The analysis window is $30 \mathrm{~m}$. $\lambda_{1}: 1558 \mathrm{~nm}$. $\Delta \lambda: 4.75 \mathrm{~nm}$. $\Delta z: 10 \mathrm{~m}$.

In general, all these effects are stronger in the case of a DSF than a standard fiber, mainly due to its smaller effective area, smaller chromatic dispersion, and larger residual birefringence [9], [10]. These aspects are clearly seen in our result, where the spatial resolution in the DSF is several times larger than the case of standard fibers. In the case of DSFs, we sometimes experienced the disappearance of the signal when we attempted to increase the spatial resolution by increasing $\Delta \lambda$. We confirmed that this effect was due to the polarization misalignment of FWM-pump waves not only within the measured fiber, but also within the HNF used for Brillouin-pump generation, which prevented us from getting a clear Brillouin-pump pulse.

Although the other factors need further investigation for improvement, we believe that the spontaneous Brillouin scattering of the FWM pumps could be suppressed by proper dithering of the optical frequency of FWM-pump waves, in such a way that the generated FWM probe would still have a narrow linewidth [11].

An important point is the dynamic range of the measurement. The dynamic range is in fact related to the obtained spatial resolution, since improving the resolution (by increasing $\Delta \lambda$ ) results in a smaller FWM signal in agreement with (2). Although detailed tests need to be done, we believe that a dynamic range in the order of $10 \mathrm{~km}$ is directly achievable for the case of the highest spatial resolution that we have achieved (around $20 \mathrm{~m}$ ). If the power of the FWM-probe wave could be increased up to the level of usual BOTDA systems (on the order of a milliwatt) by the suppression of the spontaneous Brillouin scattering of the FWM-pump waves, we believe that the range may be extended up to $\sim 30 \mathrm{~km}$, as in usual BOTDA systems [12].

\section{CONCLUSION}

We have demonstrated a high-resolution mapping of chromatic dispersion in optical fibers based on four-wave mixing (FWM) and Brillouin-optical-time-domain analysis (BOTDA) using a novel experimental scheme. With this new method, record spatial resolutions of $20 \mathrm{~m}$ in a standard fiber and $150 \mathrm{~m}$ in a dispersion-shifted fiber (DSF) were achieved, respectively. We expect that further improvement will be possible by suppressing spontaneous Brillouin scattering of the FWM pumps. This high-resolution information of chromatic dispersion will be useful in several applications, including parametric amplification and dispersion management in optical communication systems.

\section{REFERENCES}

[1] L. F. Mollenauer, P. V. Mamyshev, J. Gripp, M. J. Neubelt, N. Mamysheva, L. Gruner-Nielsen, and T. Veng, "Demonstration of massive wavelength-division multiplexing over transoceanic distances by use of dispersion-managed solitons," Opt. Lett., vol. 25, no. 10, pp. 704-706, May 2000.

[2] F. Yaman, Q. Lin, S. Radic, and G. P. Agrawal, "Impact of dispersion fluctuation on dual-pump fiber-optic parametric amplifiers," IEEE Photon. Technol. Lett., vol. 16, no. 5, pp. 1292-1294, May 2004.

[3] L. Provino, A. Mussot, E. Lantz, T. Sylvestre, and H. Maillotte, "Broadband and flat parametric amplifiers using a multi-section dispersiontailored nonlinear fiber arrangement," J. Opt. Soc. Amer. B, Opt. Phys., vol. 20, no. 7, pp. 1532-1537, Jul. 2003.

[4] L. F. Mollenauer, P. V. Mamyshev, and M. J. Neubelt, "Method for facile and accurate measurement of optical fiber dispersion maps," Opt. Lett., vol. 21, no. 21, pp. 1724-1726, Nov. 1996.

[5] M. Eiselt, R. M. Jopson, and R. H. Stolen, "Nondestructive positionresolved measurement of the zero-dispersion wavelength in an optical fiber," J. Lightw. Technol., vol. 15, no. 1, pp. 135-143, Jan. 1997.

[6] M. Gonzalez-Herraez and L. Thevenaz, "Simultaneous position-resolved mapping of chromatic dispersion and Brillouin shift along single-mode optical fibers," IEEE Photon. Technol. Lett., vol. 16, no. 4, pp. 1128-1130, Apr. 2004.

[7] K. Nakajima, M. Ohashi, and M. Tateda, "Chromatic dispersion distribution measurement along a single-mode optical fiber," J. Lightw. Technol., vol. 15, no. 7, pp. 1095-1101, Jul. 1997.

[8] M. Nikles, L. Thévenaz, and P. Robert, "Brillouin gain spectrum characterization in single-mode optical fibers," J. Lightw. Technol., vol. 15, no. 10 , pp. 1842-1851, Oct. 1997.

[9] Q. Lin and G. P. Agrawal, "Impact of polarization-mode dispersion on measurement of zero-dispersion wavelength through four-wave mixing," IEEE Photon. Technol. Lett., vol. 15, no. 12, pp. 1719-1721, Dec. 2003.

[10] M. Wuilpart, P. Megret, M. Blondel, A. J. Rogers, and Y. Defosse, "Measurement of the spatial distribution of birefringence in optical fibers," IEEE Photon. Technol. Lett., vol. 13, no. 8, pp. 836-838, Aug. 2001.

[11] S. Yamashita and M. Tani, "Cancellation of spectral spread in SBSsuppressed fiber wavelength converters using a single phase modulator," IEEE Photon. Technol. Lett., vol. 16, no. 9, pp. 2096-2098, Sep. 2004.

[12] X. Bao, D. J. Webb, and D. A. Jackson, "32-km distributed temperature sensor using Brillouin loss in optical fiber," Opt. Lett., vol. 18, no. 18, pp. 1561-1563, Sep. 1993. 


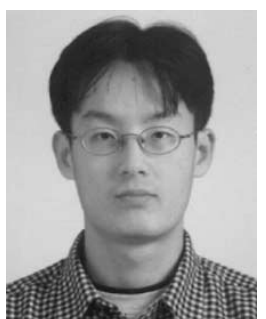

Kwang-Yong Song received the B.S., M.S., and $\mathrm{Ph} . \mathrm{D}$. degrees in physics from Korea Advanced Institute of Science and Technology (KAIST), Daejeon, South Korea, in 1996, 1998 and 2003, respectively.

In 1997, he joined the Fiber-Optics Laboratory at KAIST, where he developed fused-type fiber devices based on the fiber-taper technique for his doctoral research. In 2003, he worked as a Postdoctoral Researcher in KAIST and then moved to the Nanophotonics and Metrology Laboratory, Ecole Polytechnique Federale de Lausanne (EPFL), Switzerland, where he made researches in stimulated Brillouin scattering in optical fibers as an Academic Host. In 2005, he joined the Department of Electronic Engineering, University of Tokyo, Tokyo, Japan, as a Research Fellow. His current research interest is the application of distributed sensing in optical fibers based on Brillouin-optical-time-domain analysis (BOTDA) and Brillouin-optical-correlation-domain analysis (BOCDA).

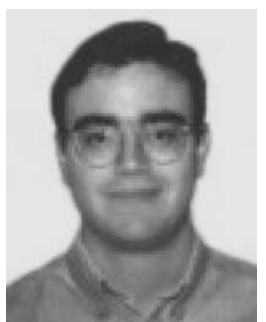

Miguel González Herráez received the M.Sc and $\mathrm{Ph} . \mathrm{D}$. degrees from the Polytechnic University of Madrid, Madrid, Spain, in 2000 and 2004, respectively.

While pursuing the $\mathrm{Ph} . \mathrm{D}$. degree, he worked as a Research Assistant first, and a Post-Doctoral Fellow later, in the Applied Physics Institute at the Spanish Council for Research, and had several long stays in the Nanophotonics and Metrology Laboratory, Ecole Polytechnique Federale de Lausanne (EPFL), Switzerland. In October 2004, he was appointed Assistant Professor in the Department of Electronics, University of Alcala, Madrid. He is the author or coauthor of over 30 papers in international refereed journals and conference contributions. His research interests cover the wide field of nonlinear interactions in optical fibers.

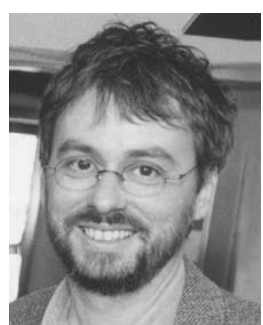

Luc Thévenaz received the M.Sc. degree in astrophysics from the Observatory of Geneva, Switzerland, in 1982, and the Ph.D. degree in physics from the University of Geneva, in 1988, which is when he developed his field of expertise, i.e., fiber optics and optical signal processing.

In 1988, he joined the Swiss Federal Institute of Technology of Lausanne (EPFL), where he currently leads a research group involved in photonics, namely fiber optics and optical sensing. His research topics include electrical-current fiber sensors, Brillouinscattering fiber sensors, fiber-nonlinearities-measurement techniques, and laser-diode (LD) spectroscopy of gases. In 1991, he visited Pontifícia Universidade Católica (PUC) University, Rio de Janeiro, Brazil, and stayed at Stanford University, Stanford, CA, where he participated in the development of a Brillouin-laser gyroscope. In 1998 and 1999, he stayed at the Korea Advanced Institute of Science and Technology (KAIST), Daejeon, South Korea, where he worked on fiber-laser current sensors. In 2000, he cofounded the company Omnisens, which is developing and commercializing advanced photonic instrumentation. 